\title{
Random tree algorithm-based pediatric pneumonia screening using heart rate, respiration rate and temperature Sumiyakhand Dagdanpurev ${ }^{1,2}$, Udval Tsogzolmaa ${ }^{1}$, Guanghao Sun $^{3}$, Lodoiravsal Choimaa ${ }^{2}$, Yukiya Hakozaki $^{4}$, Khishigmaa Baatar ${ }^{5}$, Takemi Matsui ${ }^{1 *}$
}

\author{
${ }^{1}$ Graduate School of Systems Design, Tokyo Metropolitan University, Tokyo, Japan \\ ${ }^{2}$ Machine Intelligence Laboratory, National University of Mongolia, Ulaanbaatar, Mongolia \\ ${ }^{3}$ Graduate School of Informatics and Engineering, University of Electro-Communications, Tokyo, Japan \\ ${ }^{4}$ Genkikai Yokohama Hospital, Yokohama, Japan \\ ${ }^{5}$ Bayangol Health Center, Ulaanbaatar, Mongolia
}

\begin{abstract}
Despite a reduction in pneumonia-related mortality, pneumonia remains a leading cause of death among children aged 0-5 years. Most of these deaths occur in developing countries. However, more than half of pneumonia-related deaths are preventable with improved facilities and health strategies. Early rapid diagnosis is important to decrease pneumonia mortality.

We developed a portable, cost-effective, rapid pneumonia screening system using a random tree algorithm to support early detection of pneumonia in children.

We enrolled 105 participants: 57 patients aged 1-13 years (33 boys, 24 girls) diagnosed with pneumonia by chest radiograph and 48 normal volunteers aged 2-14 years (25 boys, 22 girls). We conducted a clinical trial in the Bayangol District Geriatric and Pediatric Hospital, Ulaanbaatar, Mongolia from January 12-19, 2019. Our screening system measured heart rate, respiration rate and skin temperature within 10 seconds and used a random tree algorithm to distinguish patients with pneumonia and normal volunteers. The system uses a photosensor, Doppler radar, and infrared thermophile to determine vital signs and an Arduino Nano microprocessor to perform computations. Paired t-tests were used to compare vital signs between patients with pneumonia and normal volunteers.

The random tree algorithm achieved sensitivity of $96.5 \%$, specificity of $81.3 \%$, positive predictive value of $85.9 \%$, and negative predictive value of $95.1 \%$. The paired t-tests showed strong statistically significant differences in all three vital signs between patients with pneumonia and normal volunteers.

Our random tree algorithm-based screening system offers an effective, rapid, and convenient tool for early detection of pneumonia in children. Its cost-effectiveness enables application in low-income countries. The system measures multiple vital signs simultaneously within 10 seconds, which may be useful for initial physical examinations in pediatric hospitals.
\end{abstract}

Keywords: Pediatric, Pneumonia detection, Rapid screening, Vital signs, Random tree algorithm

\section{Introduction}

Pneumonia is a major health issue that is responsible for significant mortality and morbidity among children under 5 years [1]. The United Nations Children's Fund estimated that, worldwide, around 3 million pediatric deaths are caused by pneumonia each year [2]. Although $98 \%$ of these fatalities occur in industrialized countries, pneumonia remains a significant cause of morbidity in low-income countries with poor clinical facilities [3]. Pneumonia diagnosis is based on the patient's clinical signs based on the patient's chest radio -graph and laboratory tests [4].

Corresponding Author: Sumiyakhand Dagdanpurev, Graduate School of Systems Design, Tokyo Metropolitan University, Asahigaoka 6-6, Hino Tokyo 191-0065, Japan Received date: April 20, 2019; Accepted date: April 29, 2019; Published date: April 30, 2019.

DOI: https://doi.org/10.31546/JBRCI.1003 
These diagnosis methods are time-consuming and require good health facilities with adequate staffing. A lack of hightechnology equipment and human resources leads to delayed diagnosis, meaning pneumonia continues to be a significant health issue in developing countries. In 1980, the World Health Organization developed a pneumonia control algorithm appropriate for countries with limited facilities, which detected pneumonia based on fast breathing and lower chest indrawing [5].

A country that has a high burden from pediatric pneumonia is Mongolia, a developing country in East Asia. Every winter, hospitals in Ulaanbaatar, Mongolia's capital city, are overloaded with pediatric patients with pneumonia and influenza. This situation places a critically high workload on pediatricians and nurses. In Mongolia, children first go to their local hospital for initial screening by their family doctor. If the child is diagnosed with potential pneumonia, the doctor sends them to a next stage regional hospital for further diagnosis [6]. Regional hospital pediatricians diagnose pneumonia using chest radiography, which is highly settingdependent. During the winter, these hospitals are crowded, and children often have long wait because of the limited radiographic equipment available in regional hospitals. Therefore, parents and pediatricians prefer children to be diagnosed as early as possible by their family doctor.

We previously developed various screening systems for infectious diseases and major depressive disorder that can be used in various applications (e.g., mass screening and clinical use in Japan, Mongolia, and Vietnam) [7-9]. In this study, we developed a portable pediatric pneumonia screening system using a random tree algorithm to provide convenient, rapid, and high-accuracy pneumonia detection in children. The system is equipped with three simple biosensors (photosensor, Doppler radar, and infrared thermophile) for heart rate (HR), respiration rate $(\mathrm{RR})$, and skin temperature $(\mathrm{T})$ observation. Physical signals from the sensors are processed and numerical vital signs are calculated by software that was developed based on LabVIEW software. After determination of vital signs, patients are classified as potential pneumonia cases or normal by the random tree algorithm, which is a simple and easy to implement classification method [10]. The whole process takes 10 seconds and is performed by a low-cost microprocessor (Arduino Nano). The cost-effectiveness of the system means that it is feasible for low-income countries. The system operation is also user-friendly, meaning medical skills and experience is not required.

\section{Materials and Methods}

\section{Pediatric pneumonia screening system using HR, RR and T}

Examination of the systematic inflammatory response syndrome associated with pneumonia is routinely used in clinical practice [11]. Diagnostic criteria are fast breathing, fever, tachypnea, and white blood cell count to detect systematic inflammation in the human body [12-14]. Our proposed system measures HR, RR, and T within 10 seconds. After measurement of the numerical values of these vital signs, a random tree model distinguished potential pneumonia patients and normal volunteers. The system is designed to be placed on neck area (Fig. 1a, b), and has three biosensors (Fig. 1c) to measure vital signs. HR is measured from the carotid artery by a pulse or photoplethysmography (PPG) sensor (SEN-11574, SparkFun Electronics). PPG sensors send lowintensity infrared green light to the skin and detect light absorbed by tissues and blood. The heart beat causes blood vessel volume changes, and the light absorption varies proportionally to the blood flow change, allowing HR to be calculated [15]. A Doppler radar (NJR4262, New Japan Radio Co. Ltd.) works similarly to a PPG sensor, but radiates a 24 $\mathrm{GHz}$ microwave signal and measures RR based on detecting the reflected signal. The radar uses the Doppler effect that occurs when there is a frequency change between radiated and reflected signals because of chest movement from the breathing process [16]. $\mathrm{T}$ is measured from the neck skin by an infrared thermometer (MLX90614, Melexis NV). Infrared technology uses infrared emissions from the human skin, which enables simple, rapid, and noncontact temperature measurement [17]. Physical signals are processed from these biosensors and calculated as numerical values by an Arduino Nano microprocessor with software that was developed based on LabVIEW graphical user interface software (National Instruments, Texas, USA). Our system measures vital signs for 10 seconds, after which the resulting numerical values are shown on an LCD display, and the pneumonia screening result is indicated by a LED light. If the result is potential pneumonia, the LED light blinks green.

No medical experience or skill is required to use this system. From the start to end of measurement, the whole process is automatic. Therefore, the system is not limited to hospitals, but can be applied in homes, 
offices, and schools. In addition, measuring three vital signs simultaneously within 10 seconds may reduce clinicians' workload and simplify initial pediatric physical examinations.

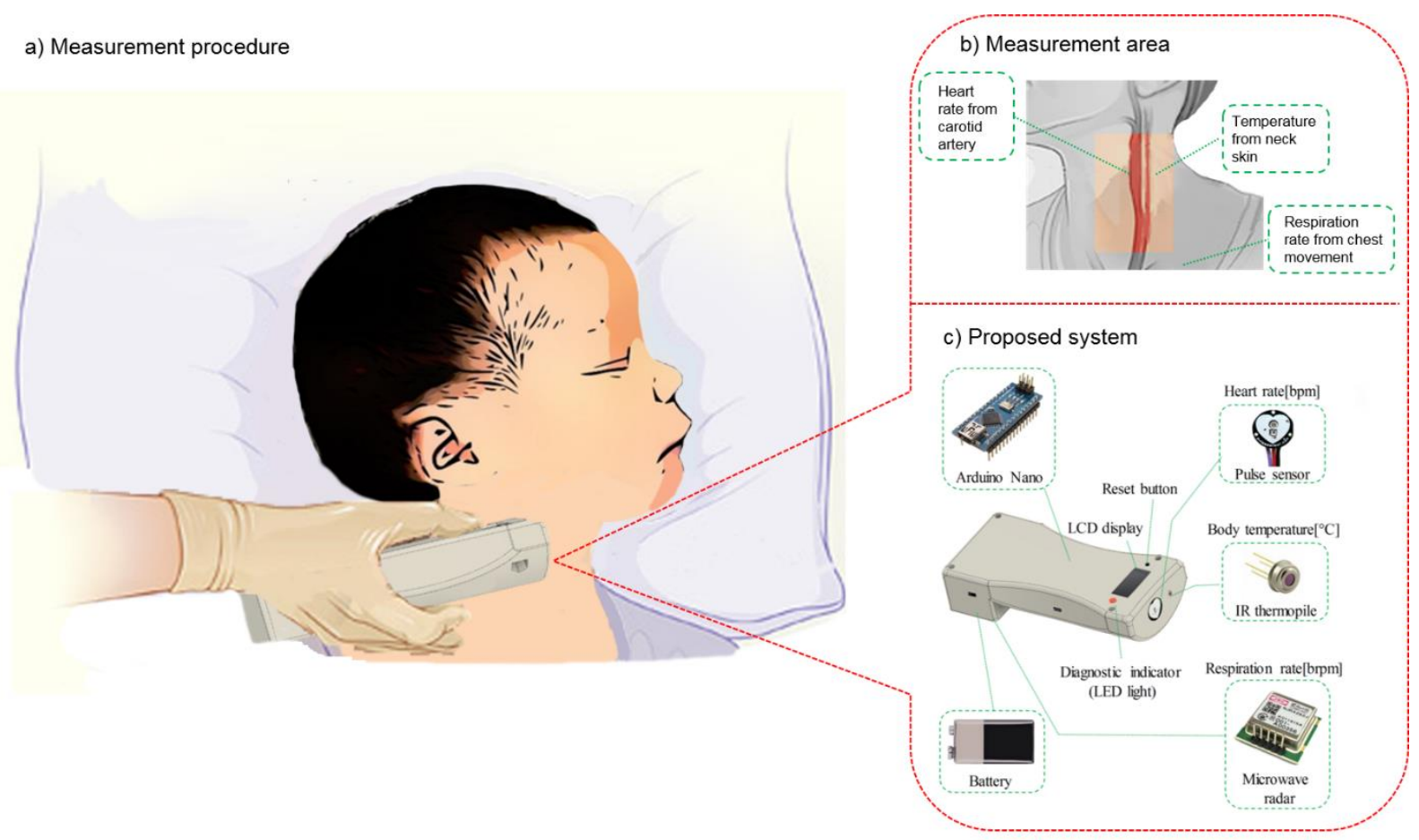

Figure 1: Measurement procedure and system design

\section{Clinical trial}

We conducted a clinical trial in the Bayangol District Geriatric and Pediatric Hospital in Ulaanbaatar, Mongolia, from January 12-19, 2019. In total, 105 children participated in the trial: 57 patients with pneumonia (based on chest radiography by a pediatrician) aged $0-13$ years (33 boys, 24 girls) and 48 normal volunteers without pneumonia symptoms aged $2-14$ years ( 25 boys, 22 girls). Children aged over 2 years were measured in a stable sitting position on a chair. We measured children younger than 2 years by laying them on an examination bed (Fig. 1b). After the doctor's physical examination, we measured $\mathrm{HR}, \mathrm{RR}$, and $\mathrm{T}$ for 10 seconds. The children's vital signs and oxygen saturation (SpO2) were measured simultaneously by usual clinical methods as a reference. The reference HR, RR, and T were measured by pulse oximeter (SAT-2200 Oxypal mini, Nihon Kohden Corporation), doctor's count, and digital thermometer (TO-401, Dretec Co., Ltd.), respectively. This study was approved by the Ethics Committee of Tokyo Metropolitan University. All parents of participants provided written consent forms.

\section{Random tree algorithm}

The random tree algorithm is a simple machinelearning algorithm. Similar to standard decision tree methods, this algorithm uses already known learning datasets to build a tree-like model that consists of sequential tests on variables (HR, RR, and $\mathrm{T}$ ) to perform binary classification [18]. A random tree algorithm is quick to implement because the algorithm chooses variables randomly to construct the tree. Previously, we used a random tree algorithm for influenza detection [19]. In addition, random tree algorithms have recently been used in various fields (e.g., industry, space, and medicine) because implementation is easy and rapid [20].

\section{Results and Discussion}

Figure 2 shows the t-test results for the comparison of vital signs between patients with pneumonia and normal volunteers, which were all statistically significant $(\mathrm{p}<0.0001)$. The $\mathrm{RR}$, which is the most important symptom of pneumonia diagnosis, was $31.5 \pm 8.3 \mathrm{brpm}$ for patients with pneumonia 
compared with $23.8 \pm 7.2$ brpm for normal volunteers [21,22]. The mean HRs for patients with pneumonia and normal volunteers were $103.2 \pm 26.9 \mathrm{bpm}$ and $82.6 \pm 13.5 \mathrm{bpm}$, respectively. There were also statistically significant differences in $\mathrm{T}$ between patients with pneumonia $(35.8 \pm 0.9$ ${ }^{\circ} \mathrm{C}$ ) and normal volunteers $\left(34.8 \pm 0.6{ }^{\circ} \mathrm{C}\right)$. The random tree model, created using Weka 3.8.1 machine-learning open source software, could distinguish patients that potentially had pneumonia and normal volunteers using three vital signs (HR, RR, and T) gathered from the 105 participants (Fig. 3). We performed the leave-one-out method to accurately evaluate system performance. The classification results showed the system had a sensitivity of $96.5 \%$, specificity of
$81.3 \%$, positive predictive value of $85.9 \%$, and negative predictive value of $95.1 \%$. Low level oxygen saturation $(\mathrm{SpO} 2<95 \%)$ in blood is a clinical sign caused by respiratory disorders, including pneumonia [23]. Therefore, we compared the $\mathrm{SpO} 2$ percentage measured by pulse oximetry simultaneously to the system measurement with the random tree algorithm performance (Fig. 4). In total, 29 of the 57 patients with pneumonia had a $\mathrm{SpO} 2$ above $95 \%$, the proposed system achieved higher performance than investigation on $\mathrm{SpO} 2$ level.

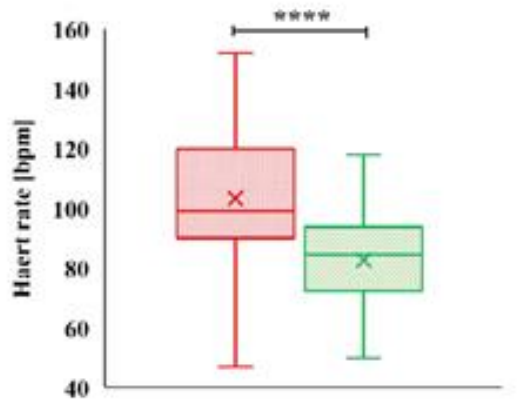

$\square$ Pneumonia patients $₫$ Normal volunteers

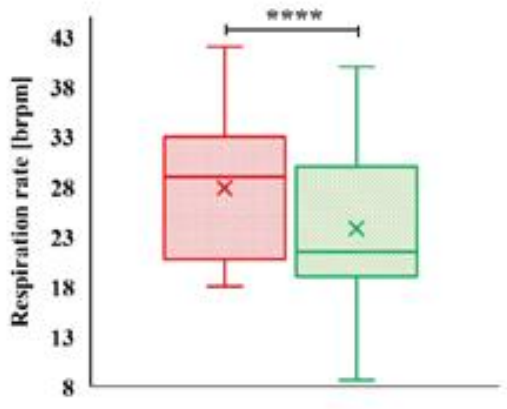

Pneumonia patients $\mathbb{Q}$ Normal volunteers

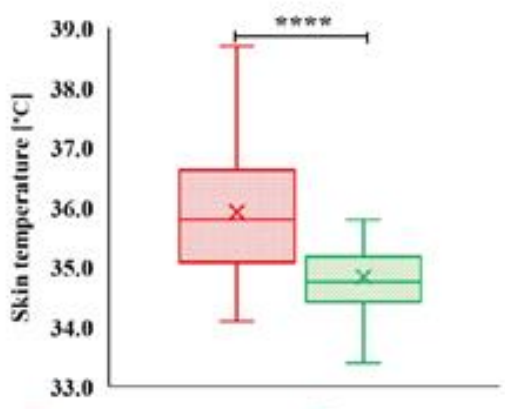

Pneumonia patients $\mathbb{Q}$ Normal volunteers

Figure 2: T-test results for patients with pneumonia and normal volunteers

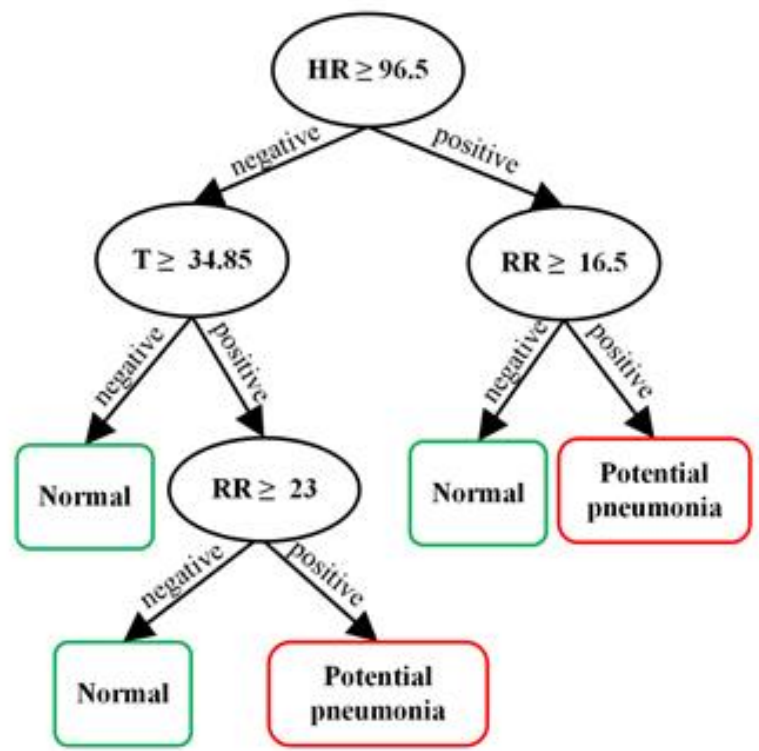

Figure 3: Random tree model built using data for 105 participants 


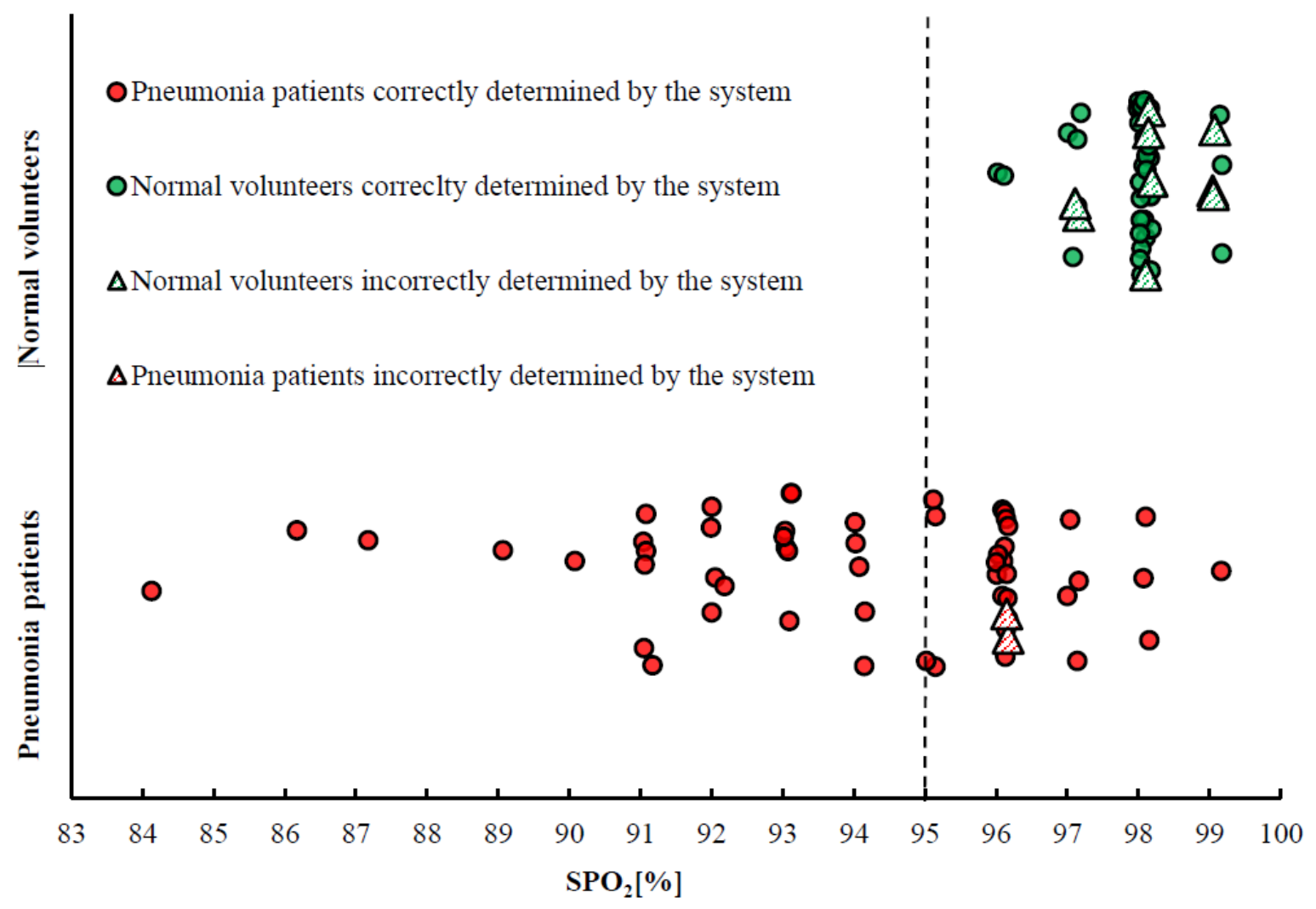

Figure 4: Classification results distinguished by the random tree algorithm

At Mongolian regional hospitals, conventional diagnosis (including chest radiography examination) of pneumonia in pediatrics takes 4-24 hours depending on the hospital workload. Our proposed system took 10 seconds to predict potential pneumonia in children.

A limitation of this study was that the system displayed vital signs without saving the physical signals and numerical values. From an engineering and system development perspective, the system should save these measurement results. We are planning to add Bluetooth connectivity to the system to allow transfer of information to personal computers or smartphones. In further studies, we will also enroll adults and older adults to broaden the system application and evaluate the random tree algorithm performance in a broad age range.

\section{Conclusion}

Pneumonia is a major cause of mortality in children under 5 years mostly in developing countries. Lack of diagnostics equipment and human resources leads to delayed treatment and morbidity. To help developing countries with the early detection of pneumonia in children, we developed a random tree algorithm-based pneumonia screening system using multiple vital signs. This system is equipped with three biosensors and can distinguish potential pneumonia patients within 10 seconds. A cost-effective microprocessor (Arduino Nano) calculates physical signals and performs the computation. Overall, the system cost is favorable for low-income countries. However, hospitals in developed countries that are equipped with hightechnology equipment for simple vital sign measurements and industrialized countries often lack human resources and devices. Therefore, our proposed system may facilitate hospitals in developing countries such as Mongolia and help pediatricians and nurses with time-consuming, complicated physical examinations as it measures multiple vital signs simultaneously in a short time.

\section{Conflict of Interest}

The authors have no conflicts of interest to declare.

Funding: None 


\section{Acknowledgements}

The authors would like to thank Dr. Soyolmaa D. for her help conducting a clinical trial.

\section{References}

1. World Health Organization. Children: reducing mortality. 2018.

2. Bennett NJ, Domachowske J, Steele RW. Pediatric pneumonia. Pediatrics: General Medicine. 2018.

3. Duke T, et al. Improved oxygen systems for childhood pneumonia: a multihospital effectiveness study in Papua New Guinea. Lancet. 2008; 372: 1328-1333.

4. Franquet T. Imaging of pneumonia: trends and algorithms. Eur Respir J. 2001; 18:196-208.

5. World Health Organization. Department for Maternal, Newborn, Child and Adolescent Health. Optimal use of clinical signs for diagnosis and prognosis of childhood pneumonia. 2018.

6. UNICEF. Mongolia air pollution crisis. 2018.

7. Sun G, et al. Field evaluation of an infectious disease/fever screening radar system during the 2017 dengue fever outbreak in Hanoi, Vietnam: a preliminary report. J Infect. 2017; 75(6):593-595.

8. Matsui T, et al. Development of a non-contact screening system for rapid medical inspection at a quarantine depot using a laser Doppler blood-flow meter, microwave radar and infrared thermography. J Med Eng Techn. 2009; 33:403-409.

9. Dagdanpurev $\mathrm{S}$, et al. Development and clinical application of a novel autonomic transient response-based screening system for major depressive disorder using a fingertip photoplethysmographic sensor. Front Bioeng Biotechnol. 2018; 6:64.

10. Kalmegh SR. Comparative analysis of WEKA data mining algorithm RandomForest, RandomTree and LADTree for classification of indigenous new data. Int $\mathbf{J}$ Emerg Techn Adv Eng. 2015; 5(1): 507-517.

11. Falup-Pecurariu OG, et al. Clinical and laboratory features of children with community-acquired pneumonia are associated with distinct radiographic presentations. Eur J Pediatr. 2018; 177(7):1111-1120.

12. Moore $\mathrm{M}$, et al. Predictors of pneumonia in lower respiratory tract infections: $3 \mathrm{C}$ prospective cough complication cohort study. Eur Resp J. 2017; 50:1700434.

13. Fleming $\mathrm{S}$, et al. Normal ranges of heart rate and respiratory rate in children from birth to 18 years of age: a systematic review of observational studies. Lancet. 2011; 377:1011-1018.
14. Daymont C, Bonafide CP, Brady PW. Heart rates in hospitalized children by age and body temperature. Pediatrics. 2015; 135(5): e1173e1181.

15. Lin WH, Wu D, Li C, Zhang $\mathrm{H}$, Zhang YT. Comparison of heart rate variability from PPG with that from ECG. The International Conference on Health Informatics. IFMBE Proceedings, 2014; 42:213-215.

16. Sun G, et al. Vital-SCOPE: Design and evaluation of a smart vital sign monitor for simultaneous measurement of pulse rate, respiratory rate, and body temperature for patient monitoring. J Sen. 2018; Article ID 4371872.

17. Dagdanpurev S, Sun G, Choimaa L, Abe S, Matsui T. Clinical application of multiple vital signs-based infection screening system in a Mongolian hospital: Optimization of facial temperature measurement by thermography at various ambient temperature conditions using linear regression analysis. Conf Proc IEEE Eng Med Biol Soc. 2018; 5313-5316.

18. Mishra AK, Ratha BK. Study of random tree and random forest data mining algorithms for microarray data analysis. IJAECE. 2016; 3(4):57.

19. Dagdanpurev S, et al. A novel machine-learningbased infection screening system via 2013-2017 seasonal influenza patients' vital signs as training datasets. J Infect. 2019; 78:409-421.

20. Zhao Y, Zhang Y. Comparison of decision tree methods for finding active objects. A S R. 2008; 41(12): 1955-1959.

21. Chang AB, Ooi MH, Perera D, Grimwood K. Improving the diagnosis, management, and outcomes of children with pneumonia: where are the gaps? Front Pediatr. 2013: 1:29.

22. Silvetti MS, Drago F, Ragonese P. Heart rate variability in healthy children and adolescents is partially related to age and gender. Int J Cardiol. 2001; 8: 169-174.

23. Duke T, Mgone J, Frank D. Hypoxaemia in children with severe pneumonia in Papua New Guinea. Int J Tuberc Lung Dis. 2001; 5(6): 511519. 